\title{
SÍFILIS CONGÊNITA NO BRASIL: PANORAMA ATUALIZADO DA INCIDÊNCIA E FATORES DE INFLUÊNCIA
}

\author{
Congenital Syphilis in Brazil: Updated Overview of Incidence and Influencing Factors
}

\author{
Beatriz Dias Freitas ${ }^{1}$ \\ Karoliny Marques do Carmo Santana ${ }^{2}$ \\ Natália Lopes de Freitas ${ }^{3}$ \\ Joel Antônio Cordeiro de Abreu ${ }^{4}$ \\ Fabiana Brandão ${ }^{5}$
}

\begin{abstract}
Resumo: Objetivo: destacar fatores de risco e discutir o panorama atual da Sífilis congênita no Brasil. Métodos: trata-se de uma revisão integrativa de literatura, baseada no modelo PRISMA com busca de artigos nas bases de pesquisas biomédicas Pubmed e Science Direct, em um intervalo de publicação de 2011 a 2020, o que resultou na produção de um fluxograma, seguindo as etapas de identificação, seleção e inclusão dos dados. Resultados: foi observado um aumento significativo na incidência de casos de sífilis gestacional e congênita na última década. Além disso, foi possível o levantamento de grupos que apresentam maior propensão em transmitir a sífilis congênita; sendo as mulheres com baixa escolaridade, em vulnerabilidade socioeconômica, em uso de drogas ilícitas, durante a gravidez, autodeclaradas pretas ou pardas e a alta incidência de sexo desprotegido, as casualidades que levaram ao aumento dos casos. Considerações finais: este estudo sugere possíveis falhas do sistema de saúde em oferecer um pré-natal adequado, diagnóstico assertivo de sífilis e outras ISTs, tratamento correto e campanhas de conscientização que alcance os grupos de maior propensão.
\end{abstract}

\footnotetext{
${ }^{1}$ Graduanda do curso de Farmácia pela Universidade de Brasília (UnB). Faculdade de Saúde, Universidade de Brasília, Campus Darcy Ribeiro, Asa Norte, Brasília-DF, 70910-900, Brasil. $₫$ bia382008@gmail.com. https://orcid.org/0000-0001-9300-4462.

${ }^{2}$ Graduanda do curso de Farmácia pela Universidade de Brasília (UnB). Faculdade de Saúde, Universidade de Brasília, Campus Darcy Ribeiro, Asa Norte, Brasília-DF, 70910-900, Brasil. $\square$ karoliny.marques@hotmail.com. (D) https://orcid.org/0000-0002-5172-4645.

${ }_{3}^{3}$ Graduanda do curso de Farmácia pela Universidade de Brasília (UnB). Faculdade de Saúde, Universidade de Brasília, Campus Darcy Ribeiro, Asa Norte, Brasília-DF, 70910-900, Brasil. $₫$ natalialopes.nlf@gmail.com. https://orcid.org/0000-0002-4814-7736.

${ }^{4}$ Mestre em Ciências Genômicas e Biotecnologia e Graduando do curso de Farmácia pela Universidade de Brasília (UnB). Faculdade de Saúde, Universidade de Brasília, Campus Darcy Ribeiro, Asa Norte, Brasília-DF, 70910-900, Brasil. $\triangle$ joelabreu1993@gmail.com. (D) https://orcid.org/0000-0002-5969-0806.

5 Pós-doutorado e Professora Adjunto do Departamento de Farmácia da. Universidade de Brasília (UnB). Laboratório de Análises Clínicas, Departamento de Farmácia, Faculdade de Saúde, Universidade de Brasília, Campus Darcy Ribeiro, Asa Norte, Brasília-DF, 70910-900, Brasil.. $₫$ fabianabrandao@unb.br. https://orcid.org/0000-0001-8358-8062.
}

$\begin{array}{ccc}\text { Recebido em } & \text { Aceito em } & \text { Publicado em } \\ 21 / 06 / 2021 & 21 / 07 / 2021 & 22 / 08 / 2021\end{array}$


Palavras-chave: Treponema pallidum; Sífilis Congênita; Incidência; Fatores de influência.

Abstract: Aim: to highlight risk factors and discuss the current situation of congenital syphilis in Brazil. Methods: integrative literature review based on the Prisma model (flowchart that includes identification, selection, and inclusion of studies) with data search on the PubMed and Science Direct websites applying the time interval from 2011 to 2020. Results: a significant increase in cases of syphilis' gestational and congenital was observed in the last decade. In addition, it was possible to point groups most prone to transmit congenital syphilis: women with low education, socioeconomic vulnerability, use of illicit drugs during pregnancy, self-declared black or brown, and high incidence of unprotected sex. Conclusion: this research evidenced failures of the health system to offer adequate prenatal care, assertive syphilis and other STI diagnosis, correct treatment, and awareness campaigns that extent groups with greater propensity.

Keywords: Treponema pallidum; Congenital syphilis; Incidence; Influencing factors

\section{INTRODUÇÃO}

A sífilis é uma Infecção Sexualmente Transmitida (IST) causada pela bactéria Treponema pallidum, subespécie pallidum, que pertence à família Treponematacea ${ }^{1-3}$. Dentre os fatores que elevam a relevância deste processo infeccioso à saúde pública, destacase o impacto na gestação, a qual causa sífilis gestacional e sífilis congênita ${ }^{4}$. Assim, os indivíduos infectados apresentam um curso da doença dividido em estágio primário, secundário, terciário e latente. Sendo os estágios primário e secundário os de maior risco de transmissão materna ${ }^{2}$.

A sífilis congênita resulta da transmissão materna de T. pallidum ao feto por via transplacentária, após a presença de espiroquetas no sangue, chamada de espiroquetemia materna $^{5,6}$. Embora a transmissão ao recém-nascido possa ocorrer, também, durante o parto pelo contato, em caso de lesão genital ${ }^{5-8}$.

Por outro lado, se descoberta nos primeiros três meses gestacionais e com tratamento correto, pode haver a prevenção da transmissão da sífilis gestacional para o feto ${ }^{6}$. No entanto, apesar do conhecimento das estratégias preventivas ideais, a sífilis congênita continua sendo uma das principais causas de mortalidade fetal e neonatal em todo o mundo ${ }^{7}$, associados, muitas vezes, aos grupos populacionais mais expostos e tendo como facilitadores grupos relacionados aos fatores sociodemográficos, à baixa escolaridade, à baixa renda e estado civil ${ }^{9}$. 
A sífilis gestacional afeta cerca de 1,5 milhão de gestantes por ano em todo o mundo $^{4,10}$. No Brasil, segundo os dados fornecidos pelo Boletim Epidemiológico da Sífilis (2020), em 2019, houve 61.127 casos de sífilis em gestantes (taxa de detecção de 20,8/1.000 nascidos vivos), 24.130 casos de sífilis congênita (taxa de incidência de 8,2/1.000 nascidos vivos) e 173 óbitos por sífilis congênita (taxa de mortalidade de 5,9/100.000 nascidos vivos) ${ }^{6}$.

Ademais, conforme os Indicadores de Sífilis do Departamento Doenças de Condições Crônicas e Infecções Sexualmente Transmissíveis (DCCI) do Ministério da Saúde (MS), estima-se que cerca de 236.603 mulheres foram diagnosticadas com sífilis gestacional no período de 2016 a 2020, sendo que, dentre esses, 106.240, provavelmente, evoluíram para sífilis congênita ${ }^{11}$.

Dessa forma, é possível observar que quase metade das gestantes não tratadas apresentam resultados adversos, pois essa doença pode afetar o desenvolvimento do feto e neonatos, aumentando a possibilidade de aborto, parto prematuro, malformações esqueléticas, meningite e pneumonia ${ }^{4,10}$. Outro agravante, oriundo desta patologia, é a neurossífilis, infecção no Sistema Nervoso Central (SNC), com alterações do líquido cefalorraquidiano em recém-nascidos assintomáticos ou casos mais graves, como paralisia geral progressiva ${ }^{4}$.

À vista disso, em 2016, a Organização Mundial da Saúde (OMS) lançou uma nova estratégia para lidar com as infecções sexualmente transmissíveis de 2016 a $2021^{12}$. Nesta nova abordagem é priorizada a eliminação da sífilis congênita por meio do rastreamento abrangente e tratamento de mulheres grávidas ${ }^{13}$.

Em consonância à política estratégica, proposta pela OMS, destaca-se a necessidade de estudos com foco em analisar e discutir prováveis fatores que influenciariam os índices crescentes de incidência da sífilis congênita. Ainda, o levantamento destes dados pode auxiliar na avaliação do impacto no aumento de casos no Brasil, bem como direcionar os pontos críticos que requerem melhoramento.

\section{MÉTODOS}

Este trabalho é uma revisão de literatura baseada no modelo PRISMA ${ }^{14}$. As bases de dados consultadas, para seleção dos trabalhos analisados, foram Pubmed e Science Direct. O critério de escolhas destes dois bancos de dados se deu por meio do abrangente conteúdo de produções científicas da área médica, bem como por serem mundialmente acessados. Os 
descritores empregados foram: Treponema pallidum, Syphilis, Syphilis Congenital \& Brazil, bem como, quando necessário, os descritores equivalentes na língua portuguesa.

Inicialmente, foram selecionados 20 artigos publicados entre os anos de 2011 a 2020, que em seus títulos apresentavam alguma correlação com o tema deste estudo. Após análise do conteúdo publicado, foram selecionados 13 artigos que atendiam aos critérios de inclusão, sendo estes: estudo com dados epidemiológicos no Brasil e fatores de risco relacionados à sífilis congênita. Como critério de exclusão foram retiradas publicações que traziam informações fora do intervalo de tempo estipulado, ou que não abordavam descrição de dados de incidência, prevalência e contexto da sífilis congênita.

Figura 1. Fluxograma demonstrando as etapas de Identificação, Seleção e Inclusão dos artigos pesquisados, de acordo modelo Prisma de revisão.

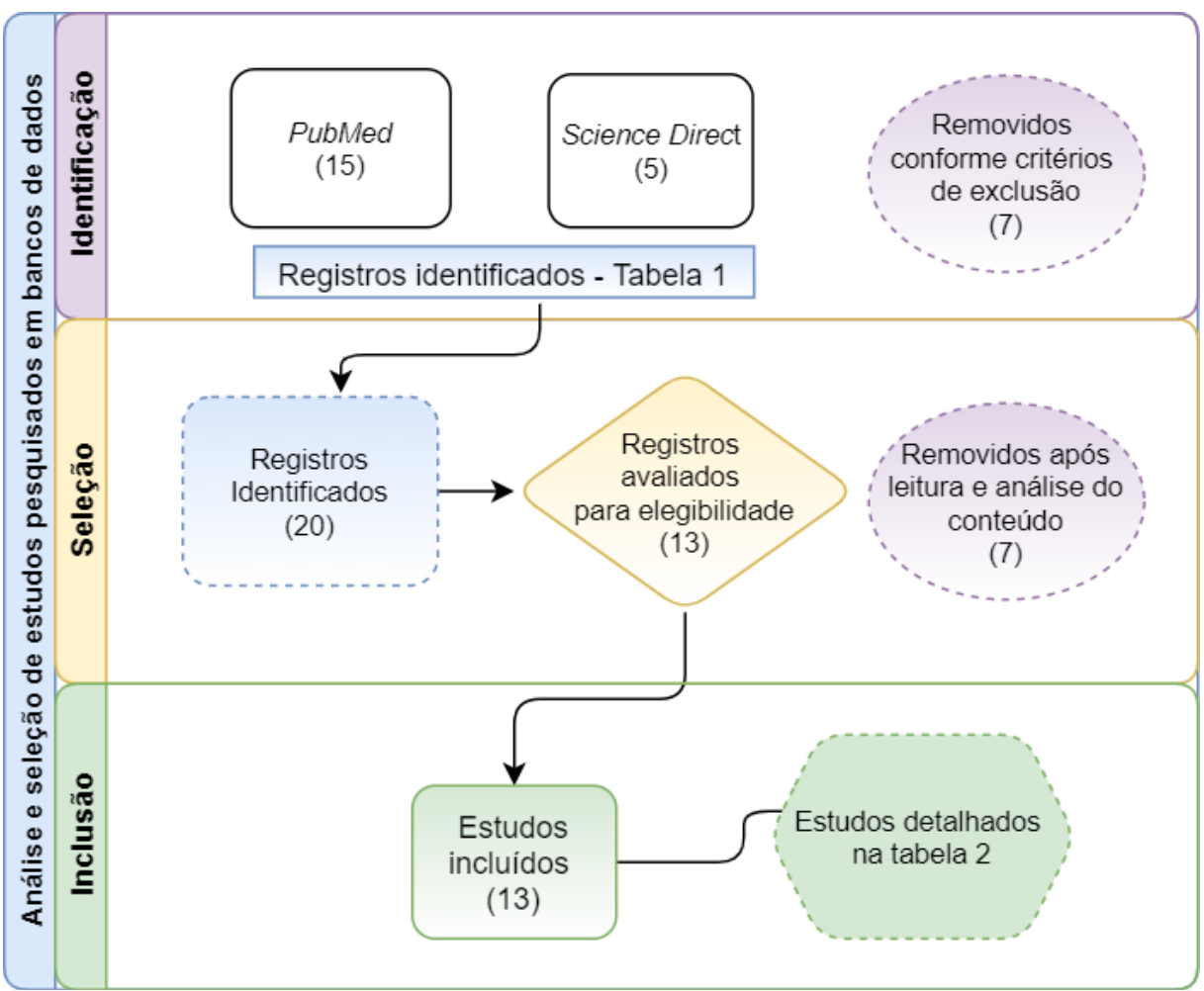

* Na etapa de Identificação sete trabalhos foram excluídos, restando 15 artigos provenientes do banco de dados PubMed e mais outros 5 provenientes do banco Science Direct. Somando todos os trabalhos detalhados na etapa 1 , chega-se ao valor de 27.

\section{RESULTADOS}

Estudos sobre a incidência de sífilis congênita no Brasil vêm alertando para um aumento exponencial na última década. Para melhor compreensão e discussão dos dados 
apontados nesta pesquisa, a aplicação dos "filtros" que resultaram na seleção e inclusão dos artigos pesquisados, foram organizados em duas tabelas detalhadas a seguir.

Os dados apresentados na Tabela 1 foram agrupados conforme a etapa de seleção e descreve informações gerais dos treze artigos selecionados. Nesta etapa, foram analisados título, categoria do estudo, autores, local e data dos estudos. Foi possível observar que em alguns estudos não foram identificados dados de localidade em que foi feita a pesquisa e nem o intervalo de tempo analisado.

Tabela 1 - Informações gerais dos artigos da etapa Seleção.

\begin{tabular}{|c|c|c|c|c|c|}
\hline Título do artigo & $\begin{array}{l}\text { Categoria de } \\
\text { artigo }\end{array}$ & Autores & Estado/País & Ano de estudo & Referência \\
\hline $\begin{array}{c}\text { Incidência de } \\
\text { sífilis congênita e } \\
\text { fatores } \\
\text { associados à } \\
\text { transmissão } \\
\text { vertical da sífilis: } \\
\text { dados do estudo } \\
\text { Nascer no Brasil }\end{array}$ & $\begin{array}{l}\text { Estudo nacional } \\
\text { de base hospitalar }\end{array}$ & $\begin{array}{l}\text { Rosa Maria Soares } \\
\text { Madeira Domingues, } \\
\text { Maria do Carmo Leal }\end{array}$ & Brasil & 2011-2012 & 15 \\
\hline $\begin{array}{c}\text { Trends of } \\
\text { syphilis in Brazil: } \\
\text { A growth portrait } \\
\text { of the treponemic } \\
\text { epidemic }\end{array}$ & $\begin{array}{c}\text { Estudo de revisão } \\
\text { das bases oficiais } \\
\text { governamentais }\end{array}$ & $\begin{array}{c}\text { Marques Dos Santos } \\
\text { M, Lopes AKB, } \\
\text { Roncalli AG, Lima } \\
\text { KC }\end{array}$ & Brasil & $2007-2017$ & 16 \\
\hline $\begin{array}{l}\text { Prevalence of } \\
\text { syphilis in } \\
\text { pregnancy and } \\
\text { prenatal syphilis } \\
\text { testing in Brazil: } \\
\text { Birth in Brazil } \\
\text { study }\end{array}$ & Estudo de coorte & $\begin{array}{c}\text { Domingues RM, } \\
\text { Szwarcwald CL, } \\
\text { Souza Junior PR, } \\
\text { Leal Mdo C. }\end{array}$ & Brasil & 2011-2012 & 15 \\
\hline $\begin{array}{l}\text { High-risk clusters } \\
\text { and temporal } \\
\text { trends in } \\
\text { congenital } \\
\text { syphilis infection } \\
\text { in Brazil }\end{array}$ & Estudo ecológico & $\begin{array}{c}\text { Oliveira VDS, } \\
\text { Rodrigues RL, } \\
\text { Chaves VB, Dos } \\
\text { Santos TS, de Assis } \\
\text { FM, Ternes YMF, de } \\
\text { Aquino ÉC }\end{array}$ & Brasil & 2001-2017 & 17 \\
\hline $\begin{array}{l}\text { High Prevalence } \\
\text { of Syphilis and } \\
\text { Inadequate } \\
\text { Prenatal Care in } \\
\text { Brazilian } \\
\text { Pregnant } \\
\text { Women: A } \\
\text { Cross-Sectional } \\
\text { Study }\end{array}$ & $\begin{array}{l}\text { Estudo } \\
\text { transversal }\end{array}$ & $\begin{array}{l}\text { Benedetti KCSV, } \\
\text { Ribeiro ADDC, } \\
\text { Queiroz JHFS, Melo } \\
\text { ABD, Batista RB, } \\
\text { Delgado FM, da } \\
\text { Silva KE, Croda J, } \\
\text { Simionatto S. }\end{array}$ & $\begin{array}{l}\text { Mato Grosso do } \\
\text { Sul/ Brasil }\end{array}$ & 2015 & 18 \\
\hline $\begin{array}{c}\text { Mother-to-child } \\
\text { transmission and } \\
\text { gestational }\end{array}$ & $\begin{array}{c}\text { Estudo da } \\
\text { dinâmica sazonal } \\
\text { das ISTs }\end{array}$ & $\begin{array}{l}\text { Marinho de Souza J, } \\
\text { Giuffrida R, Ramos } \\
\text { APM, Morceli G, }\end{array}$ & $\begin{array}{c}\text { São Paulo (Pontal } \\
\text { do } \\
\text { Paranapanema)/ }\end{array}$ & 2011 & 4 \\
\hline
\end{tabular}




\begin{tabular}{|c|c|c|}
\hline $\begin{array}{l}\text { syphilis: Spatial- } \\
\text { temporal } \\
\text { epidemiology and } \\
\text { demographics in } \\
\text { a Brazilian region }\end{array}$ & $\begin{array}{c}\text { Coelho } \mathrm{CH} \text {, Pimenta } \\
\text { Rodrigues MV. }\end{array}$ & Brasil \\
\hline
\end{tabular}

\begin{tabular}{|c|c|c|c|c|c|}
\hline $\begin{array}{c}\text { Syphilis } \\
\text { notifications } \\
\text { among pregnant } \\
\text { women in Campo } \\
\text { Grande, state of } \\
\text { Mato Grosso do } \\
\text { Sul, Brazil, } 2011 \\
\text { to } 2017\end{array}$ & Estudo descritivo & $\begin{array}{c}\text { Pires Cássia de } \\
\text { Paula, Fernandes } \\
\text { Caroliny Oviedo, } \\
\text { Oliveira Everton } \\
\text { Falcão de, Freitas } \\
\text { Sandra Luzinete } \\
\text { Felix de, Almeida } \\
\text { Rodrigo Guimarães } \\
\text { dos Santos }\end{array}$ & $\begin{array}{l}\text { Mato Grosso do } \\
\text { Sul (Campo } \\
\text { Grande)/ Brasil }\end{array}$ & 2011-2017 & 19 \\
\hline $\begin{array}{l}\text { Late diagnosis of } \\
\text { congenital } \\
\text { syphilis: a } \\
\text { recurring reality } \\
\text { in women and } \\
\text { children health } \\
\text { care in Brazil }\end{array}$ & $\begin{array}{l}\text { Artigo original: } \\
\text { Relato de caso }\end{array}$ & $\begin{array}{l}\text { Andrade ALMB, } \\
\text { Magalhães PVVS, } \\
\text { Moraes MM, } \\
\text { Tresoldi AT, Pereira } \\
\text { RM. }\end{array}$ & São Paulo/ Brasil & $\mathrm{X}$ & 20 \\
\hline $\begin{array}{l}\text { Attributable } \\
\text { fraction of } \\
\text { congenital } \\
\text { syphilis due to } \\
\text { the lack of } \\
\text { prenatal care }\end{array}$ & Estudo ecológico & $\begin{array}{c}\text { Martinez Edson } \\
\text { Zangiacomi, } \\
\text { Zucoloto Miriane } \\
\text { Lucindo, Silva } \\
\text { Anderson Soares da, } \\
\text { Brunherotti Marisa } \\
\text { Afonso Andrade }\end{array}$ & São Paulo/ Brasil & $2010-2015$ & 21 \\
\hline $\begin{array}{l}\text { Syphilis during } \\
\text { pregnancy: a } \\
\text { preventable } \\
\text { thereat to } \\
\text { maternal- fetal } \\
\text { health }\end{array}$ & $\begin{array}{l}\text { Dados } \\
\text { governamentais }\end{array}$ & $\begin{array}{c}\text { Martha } \\
\text { W.F.RacMDaPaula } \\
\text { A.Revell } \\
\text { PhDbCatherin, } \\
\text { S.EppesMD, MPHa }\end{array}$ & Dados mundiais & $\mathrm{X}$ & 22 \\
\hline $\begin{array}{c}\text { Congenital } \\
\text { Syphilis as a } \\
\text { Measure of } \\
\text { Maternal and } \\
\text { Child Healthcare, } \\
\text { Brazil }\end{array}$ & $\begin{array}{c}\text { Abordagem } \\
\text { qualitativa de } \\
\text { estudo ecológico. }\end{array}$ & $\begin{array}{c}\text { Maria Lusia de } \\
\text { Morais Belo Bezerra, } \\
\text { Flávia Emília } \\
\text { Cavalcante Valença } \\
\text { Fernandes, João } \\
\text { Paulo de Oliveira } \\
\text { Nunes, Solma Lúcia } \\
\text { Souto Maior de } \\
\text { Araújo Baltar, Karina } \\
\text { Perrelli Randau } \\
\text { Comments to Author, } \\
\text { Karina Perrelli } \\
\text { Randau. }\end{array}$ & Brasil & $2010-2015$ & 3 \\
\hline
\end{tabular}

\begin{tabular}{|c|c|c|c|c|c|}
\hline $\begin{array}{c}\text { Molecular } \\
\text { detection of } \\
\text { Treponema } \\
\text { pallidum sp. } \\
\text { pallidum in blood } \\
\text { samples of } \\
\text { VDRL- } \\
\text { seroreactive } \\
\text { women with }\end{array}$ & $\begin{array}{l}\text { Estudo } \\
\text { retrospectivo }\end{array}$ & $\begin{array}{c}\text { Casal Charliana } \\
\text { Aragão Damasceno, } \\
\text { Silva Mayra Oliveira } \\
\text { da, Costa Igor Brasil, } \\
\text { Araújo Eliete da } \\
\text { Cunha, Corvelo } \\
\text { Tereza Cristina de } \\
\text { Oliveira }\end{array}$ & Pará/ Brasil & 2004 & 23 \\
\hline
\end{tabular}




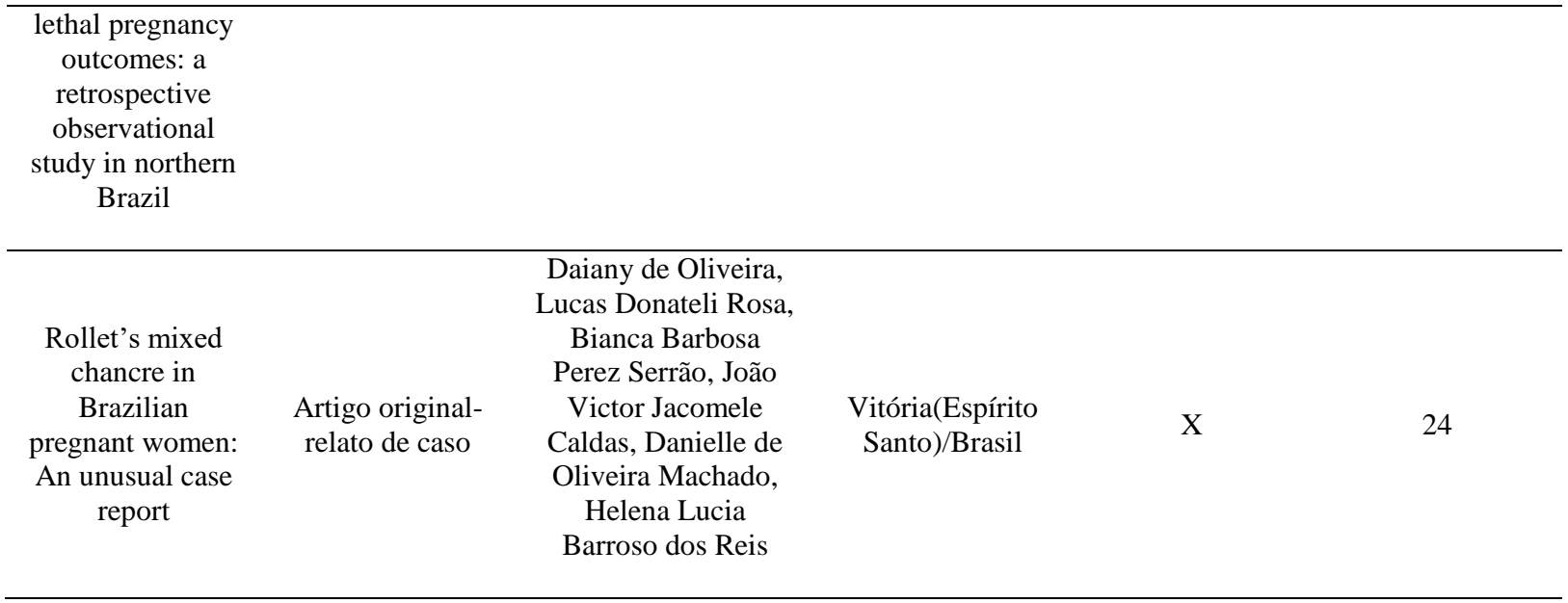

Após leitura e análise do conteúdo na fase de Seleção, seguindo os critérios de inclusão e exclusão estabelecidos, os treze artigos seguiram para última etapa do modelo de revisão PRISMA, a fase de Inclusão. A Tabela 2 foi gerada a partir destes estudos e apresenta detalhes observados na análise como os parâmetros: Fator de influência, Intervenção, Incidência e Resultados alcançados.

Tabela 2 - Informações e parâmetros analisados dos artigos analisados. Trabalhos incluídos após etapa de seleção.

\begin{tabular}{|c|c|c|c|c|c|}
\hline Título do artigo & $\begin{array}{c}\text { Fator de } \\
\text { influência }\end{array}$ & Intervenção & $\begin{array}{c}\text { Incidência dos } \\
\text { estudos }\end{array}$ & Resultados & Referência \\
\hline $\begin{array}{l}\text { Incidência de } \\
\text { sífilis congênita } \\
\text { e fatores } \\
\text { associados à } \\
\text { transmissão } \\
\text { vertical da } \\
\text { sífilis: dados do } \\
\text { estudo nascer no } \\
\text { Brasil }\end{array}$ & $\begin{array}{c}\text { Faixa etária de } 20 \text { a } \\
34 \text { anos; } \\
\text { Escolaridade } \\
\text { materna; } \\
\text { Etnia materna; } \\
\text { Pré-natal } \\
\text { inadequado; }\end{array}$ & $\begin{array}{l}\text { Para o diagnóstico } \\
\text { da sífilis na } \\
\text { gestação, foram } \\
\text { utilizados dados do } \\
\text { cartão de pré-natal } \\
\text { e do prontuário } \\
\text { hospitalar da } \\
\text { puérpera e do } \\
\text { recém-nascido. }\end{array}$ & $\begin{array}{c}\mathrm{N}^{\circ} \text { de } \\
\text { casos:18.207 } \\
\text { Taxa de } \\
\text { detecção: } 9,5 \text { no } \\
\text { ano de } 2011 \\
\mathrm{~N}^{\circ} \text { de casos: } \\
27.913 \\
\text { Taxa de } \\
\text { detecção: } 14,4 \\
\text { no ano de } 2012 \\
\end{array}$ & $\begin{array}{c}\text { A taxa de } \\
\text { transmissão vertical } \\
\text { no país foi de } \\
34,3 \% .\end{array}$ & 15 \\
\hline $\begin{array}{l}\text { Trends of } \\
\text { syphilis in } \\
\text { Brazil: A } \\
\text { growth portrait } \\
\text { of the } \\
\text { treponemic } \\
\text { epidemic }\end{array}$ & $\begin{array}{c}\text { Pré-natal } \\
\text { inadequado; } \\
\text { Tratamento tardio; }\end{array}$ & $\begin{array}{l}\text { A tendência da } \\
\text { sífilis foi analisada } \\
\text { para identificar se } \\
\text { um possível } \\
\text { aumento, } \\
\text { estabilidade ou } \\
\text { declínio ao longo } \\
\text { do período } \\
\text { estudado seria } \\
\text { significativo ou se } \\
\text { havia um efeito } \\
\text { aleatório. }\end{array}$ & $\begin{array}{c}\mathrm{N}^{\circ} \text { de casos: } \\
49.154 \text { no ano de } \\
2020 \text { no Brasil }\end{array}$ & $\begin{array}{c}\text { A taxa de sífilis } \\
\text { adquirida no Brasil } \\
\text { apresentou um } \\
\text { aumento de } 561 \% \\
\text { dos anos de } 2011 \text { a } \\
2017\end{array}$ & 10 \\
\hline $\begin{array}{l}\text { Prevalence of } \\
\text { syphilis in } \\
\text { pregnancy and } \\
\text { prenatal syphilis }\end{array}$ & $\begin{array}{c}\text { Pré-natal } \\
\text { inadequado; } \\
\text { Escolaridade } \\
\text { materna; }\end{array}$ & $\begin{array}{c}\text { O estudo foi } \\
\text { constituído por } \\
\text { mães com partos } \\
\text { hospitalares de feto }\end{array}$ & $\begin{array}{c}\mathrm{N}^{\circ} \mathrm{de} \\
\text { casos:18.207 } \\
\text { Taxa de } \\
\text { detecção: } 9,5 \text { no }\end{array}$ & $\begin{array}{c}\text { A prevalência } \\
\text { estimada de sífilis } \\
\text { na gravidez foi de } \\
1,02 \% \text {. A }\end{array}$ & 25 \\
\hline
\end{tabular}




\begin{tabular}{|c|c|c|c|c|c|}
\hline $\begin{array}{l}\text { testing in Brazil: } \\
\text { Birth in Brazil } \\
\text { study }\end{array}$ & Etnia materna; & $\begin{array}{c}\text { vivo de qualquer } \\
\text { idade gestacional } \\
\text { ou peso, ou feto } \\
\text { morto com idade } \\
\text { gestacional > } 22 \\
\text { semanas ou peso }> \\
500 \text { gramas. }\end{array}$ & $\begin{array}{c}\text { ano de } 2011 \\
\mathrm{~N}^{\circ} \text { de } \\
\text { casos:27.913 } \\
\text { Taxa de } \\
\text { detecção: } 14,4 \\
\text { no ano de } 2012\end{array}$ & $\begin{array}{c}\text { prevalência não } \\
\text { diferiu } \\
\text { significativamente } \\
\text { por região e variou } \\
\text { entre } 0,76 \% \text { na } \\
\text { região Norte e } 1,1 \% \\
\text { na região Sul }\end{array}$ & \\
\hline $\begin{array}{l}\text { High-risk } \\
\text { clusters and } \\
\text { temporal trends } \\
\text { in congenital } \\
\text { syphilis } \\
\text { infection in } \\
\text { Brazil }\end{array}$ & $\begin{array}{c}\text { Pré-natal } \\
\text { inadequado; } \\
\text { Condições } \\
\text { socioeconômicas; } \\
\text { Tratamento } \\
\text { inadequado; }\end{array}$ & $\begin{array}{l}\text { A existência de } \\
\text { aglomerados de } \\
\text { municípios } \\
\text { (clusters) com alto } \\
\text { risco para sífilis } \\
\text { congênita no Brasil } \\
\text { e descrever a } \\
\text { tendência temporal } \\
\text { da doença no país. }\end{array}$ & $\begin{array}{c}\mathrm{N}^{\circ} \text { de casos: } \\
113.806 \\
\text { Taxa de } \\
\text { detecção: } 28,4 \\
\text { dos anos de } 2001 \\
\text { a } 2017\end{array}$ & $\begin{array}{c}\text { Detectou-se uma } \\
\text { tendência crescente } \\
\text { na infecção por SC } \\
\text { em todas as regiões } \\
\text { e unidades da } \\
\text { federação. As taxas } \\
\text { foram } 8,53 \text { vezes } \\
\text { maiores nos } \\
\text { neonatos cujas mães } \\
\text { não realizaram pré- } \\
\text { natal }\end{array}$ & 17 \\
\hline
\end{tabular}

\begin{tabular}{cc}
\hline $\begin{array}{c}\text { High Prevalence } \\
\text { of Syphilis and } \\
\text { Inadequate }\end{array}$ & \\
Prenatal Care in & Tratamento tardio; \\
Brazilian & Pré-natal \\
Pregnant & inadequado; \\
Women: A & Uso de drogas; \\
Cross-Sectional & \\
Study & \\
& \\
\hline Mother-to-child & \\
transmission & Falha de \\
and gestational & diagnóstico; \\
syphilis: & Não adesão do \\
Spatial-temporal \\
epidemiology \\
and & Parceironto; não \\
demographics in & tratado; \\
a Brazilian & Pré-natal \\
region & inadequado; \\
& \\
\hline
\end{tabular}

Mulheres grávidas e seus recémnascidos foram incluídos neste estudo.

$\mathrm{N}^{\circ}$ de
casos:1.132
Taxa de
detecção: 42,7
no ano de 2015

A soroprevalência

da sífilis em

mulheres grávidas

foi de $4,4 \%$

\section{8}

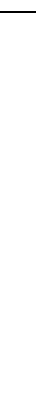




\begin{tabular}{|c|c|c|c|c|c|c|}
\hline & & & & $\begin{array}{c}\text { todas as ações } \\
\text { estabelecidas pelo } \\
\text { MS }\end{array}$ & & \\
\hline $\begin{array}{l}\text { Attributable } \\
\text { fraction of } \\
\text { congenital } \\
\text { syphilis due to } \\
\text { the lack of } \\
\text { prenatal care }\end{array}$ & $\begin{array}{c}\text { Pré-natal } \\
\text { inadequado; } \\
\text { Etnia materna; } \\
\text { baixa escolaridade; }\end{array}$ & $\begin{array}{c}\text { Determinar a } \\
\text { fração atribuível de } \\
\text { SC devido à falta } \\
\text { de pré-natal em } \\
\text { mulheres expostas } \\
\text { (AFexp) }\end{array}$ & $\begin{array}{c}\mathrm{N}^{\circ} \text { de casos: } \\
7.132 \\
\text { nos anos de } \\
2010-2015\end{array}$ & $\begin{array}{l}\text { Os principais riscos } \\
\text { para a sífilis foram } \\
\text { a baixa escolaridade } \\
\text { materna, o uso de } \\
\text { álcool e / ou drogas, } \\
\text { múltiplos parceiros } \\
\text { sexuais e baixo } \\
\text { acesso aos cuidados } \\
\text { pré-natais. }\end{array}$ & & 21 \\
\hline $\begin{array}{l}\text { Syphilis during } \\
\text { pregnancy: a } \\
\text { preventable } \\
\text { thereat to } \\
\text { maternal- fetal } \\
\text { health }\end{array}$ & $\begin{array}{l}\text { Disparidades } \\
\text { sociais } \\
\text { Condições } \\
\text { socioeconômicas } \\
\text { Etnia materna }\end{array}$ & $\begin{array}{c}\text { Disparidades } \\
\text { raciais substanciais } \\
\text { são observadas em } \\
\text { casos de sífilis } \\
\text { durante a gravidez. } \\
\text { As mulheres afro- } \\
\text { americanas } \\
\text { representam o } \\
\text { grupo demográfico } \\
\text { mais afetado. }\end{array}$ & $X$ & $\begin{array}{c}\text { As razões para o } \\
\text { aumento dos casos } \\
\text { de SC não são } \\
\text { claras, mas } \\
\text { provavelmente } \\
\text { multifatoriais. } \\
\text { Segundo dados do } \\
\text { CDC, } 21,8 \% \text { das } \\
458 \text { mulheres que } \\
\text { deram à luz bebês } \\
\text { com SC em } 2014 \\
\text { não realizaram pré- } \\
\text { natal. }\end{array}$ & & 22 \\
\hline $\begin{array}{l}\text { Congenital } \\
\text { Syphilis as a } \\
\text { Measure of } \\
\text { Maternal and } \\
\text { Child } \\
\text { Healthcare, } \\
\text { Brazil }\end{array}$ & $\begin{array}{l}\text { Qualidade da } \\
\text { atenção básica à } \\
\text { saúde materno- } \\
\text { infantil. } \\
\text { Pré-natal } \\
\text { inadequado }\end{array}$ & $\begin{array}{l}\text { Dados } \\
\text { epidemiológicos } \\
\text { agregados sobre } \\
\text { sífilis em mulheres } \\
\text { grávidas e crianças. }\end{array}$ & $\begin{array}{c}\mathrm{N}^{\circ} \text { de casos: } \\
209.211 \text { nos } \\
\text { anos de } 2010- \\
2015\end{array}$ & $\begin{array}{l}\text { Nas taxas médias de } \\
\text { sífilis congênita por } \\
\text { ano, com a maior } \\
\text { taxa média } \\
\text { registrada em } 2015 \\
(5,92 \text { / } 1.000 \\
\text { nascidos vivos). }\end{array}$ & & 3 \\
\hline $\begin{array}{c}\text { Molecular } \\
\text { detection of } \\
\text { Treponema } \\
\text { pallidum sp. } \\
\text { pallidum in } \\
\text { blood samples } \\
\text { of VDRL- } \\
\text { seroreactive } \\
\text { women with } \\
\text { lethal pregnancy } \\
\text { outcomes: a } \\
\text { retrospective } \\
\text { observational } \\
\text { study in } \\
\text { northern Brazil } \\
\end{array}$ & $\begin{array}{l}\text { Diagnóstico tardio; } \\
\text { Tratamento } \\
\text { inadequado }\end{array}$ & $\begin{array}{c}4.912 \text { mulheres } \\
\text { admitidas para } \\
\text { parto ou curetagem } \\
\text { no centro obstétrico } \\
\text { da Fundação Santa } \\
\text { Casa de } \\
\text { Misericórdia do } \\
\text { Pará }\end{array}$ & $\begin{array}{c}\mathrm{N}^{\circ} \text { de casos: } 36 \\
\text { no ano de } 2004 \\
\text { no estado do } \\
\text { Pará } \\
2004\end{array}$ & $\begin{array}{c}\text { Durante o período } \\
\text { do estudo, } 36 \\
(0,7 \%) \text { dos } 4.912 \\
\text { casos de parto e } \\
\text { curetagem eram } \\
\text { mulheres com teste } \\
\text { de triagem VDRL } \\
\text { positivo para sífilis } \\
\text { que apresentaram } \\
\text { resultado negativo } \\
\text { de gravidez. }\end{array}$ & & 23 \\
\hline $\begin{array}{l}\text { Rollet's mixed } \\
\text { chancre in } \\
\text { Brazilian } \\
\text { pregnant } \\
\text { women: An } \\
\text { unusual case } \\
\text { report }\end{array}$ & $\begin{array}{l}\text { Pré-natal } \\
\text { inadequado } \\
\text { Tratamento } \\
\text { inadequado } \\
\text { Diagnóstico } \\
\text { inadequado }\end{array}$ & $\begin{array}{l}\text { Mulher, } 19 \text { anos, } \\
\text { terceiro trimestre } \\
\text { de gestação, com } \\
\text { úlceras genitais }\end{array}$ & $X$ & $\begin{array}{l}\text { O cancro misto de } \\
\text { Rollet é a } \\
\text { associação de Sífilis } \\
\text { e cancro mole no } \\
\text { mesmo paciente. } \\
\text { Quando isso ocorre } \\
\text { na gestante, } \\
\text { representa um } \\
\text { desafio para o } \\
\text { diagnóstico e } \\
\text { tratamento } \\
\text { adequados }\end{array}$ & 24 & \\
\hline
\end{tabular}


Para além dos dados apresentados nas tabelas, sobre o Fator de influência foi feita uma análise das informações disponíveis no Departamento de Doenças de Condições Crônicas e Infecções Sexualmente Transmissíveis (DCCI) do Ministério da Saúde ${ }^{11,26}$, correlacionando com as informações descritas nos artigos analisados.

Segundo a análise dos dados incluídos neste estudo, no tocante ao Brasil, observou-se, na última década, um significativo aumento de sífilis gestacional e congênita em todas as regiões brasileiras (Norte, Nordeste, Sudeste, Sul e Centro-Oeste); sendo a região Sudeste a que apresentou maior incidência ${ }^{3,16}$.

As menores taxas de cobertura do pré-natal foram observadas entre as mulheres indígenas, as residentes na região Norte e menos escolarizadas. Já em relação às que deram à luz em unidades públicas de saúde, a qualidade da assistência pré-natal no país foi variável a depender da região, tendo relação com características socioeconômicas e demográficas ${ }^{15,17}$.

$\mathrm{Na}$ análise dos dados epidemiológicos globais, observou-se que T. pallidum causou cerca de 930.000 infecções maternas em 2012, levando a 350.000 resultados adversos da gravidez, incluindo 143.000 fetos prematuros e natimortos, 62.000 mortes neonatais, 44.000 bebês prematuros ou recém-nascidos de baixo peso ao nascer e 102.000 infecções em bebês em todo o mundo.

\section{DISCUSSÃO}

A incidência de sífilis adquirida aumentou significativamente no Brasil e a falta de diagnóstico pode ter levado a casos subnotificados de sífilis gestacional, consequentemente, resultando no aumento de casos da sífilis congênita. Destarte, os dados analisados corroboram com os achados que apontam a sífilis no período gestacional e ao nascimento, como um problema grave de saúde pública, devido à elevada incidência e internações de neonatos. Destaca-se, ainda, que houve aumento dos gastos para o sistema de saúde pública em decorrência da Sífilis congênita, sendo esta problemática completamente evitável ${ }^{15,16}$.

Houve maior incidência de casos de sífilis gestacional em mulheres autodeclaradas negras ou pardas, com baixa escolaridade, baixo índice socioeconômico e em uso de drogas ilícitas durante a gravidez. Isso, possivelmente, se deve à falta de informações sobre prevenção e tratamento e à alta incidência de sexo desprotegido entre as mulheres 
desses grupos. Este último ponto ressalta a importância dos métodos educacionais e campanhas de esclarecimento à população geral acerca da prevenção da contaminação e infecção. Muito embora se acreditava que tais métodos preventivos sejam bem conhecidos, a falha na aplicabilidade e o aumento de casos de sífilis em gestante alertam para possíveis falhas nesta etapa ${ }^{15,18,19}$.

Além disso, a cobertura de pré-natal no Brasil é insuficiente para garantir o controle da sífilis gestacional, agravando assim o diagnóstico tardio da sífilis congênita, sendo este ponto de indubitável importância para prevalência da sífilis congênita ${ }^{15,18}$.

Dados epidemiológicos indicam que a taxa de cobertura pré-natal no Brasil é menor na região Norte, particularmente entre mulheres com menor escolaridade e indígenas, possivelmente por conta das barreiras geográficas, culturais e sociais. Esse resultado indica que estratégias distintas são necessárias para alcançar populações consideradas mais vulneráveis ${ }^{15}$.

Neste contexto, as diretrizes nacionais para a sífilis congênita e gestacional determinam que a infecção deve ser rastreada duas vezes durante o pré-natal: no início da gravidez e no início do terceiro trimestre ${ }^{7}$. As mulheres devem ser reexaminadas na admissão ao parto ou quando há caso de aborto. Esta estratégia permite a identificação de mulheres com infecções não tratadas antes da concepção e aquelas infectadas durante a gravidez.

O rastreamento correto permite o tratamento adequado, evitando infecção fetal ou neonatal e prevenindo a prevalência da sífilis congênita ${ }^{5}$. É de suma importância a realização de testes treponêmicos e não treponêmicos no pré-natal, e, ainda, no momento da internação para realizar o parto, ou mesmo por curetagem pós-aborto, pois há à possibilidade de resultados falso-negativos no teste não treponêmico que antecedem esta etapa ${ }^{5,20}$.

É importante salientar que os dados de incidência e os grupos mais vulneráveis observados, neste estudo, apontou que visto que os determinantes da sífilis e da sífilis congênita não estão relacionados apenas à qualidade da atenção pré-natal, oferecida às mulheres, mas podem também estar enraizados nos fatores sociais, econômicos, culturais e comportamentais que as mulheres vivenciam essas dificuldades ${ }^{4}$.

No entanto, em desacordo aos dados mostrados, o estudo em Pontal do Paranapanema - São Paulo demonstrou maior prevalência de sífilis gestacional e congênita em mulheres autodeclaradas brancas, contrariando estudos anteriores de maior prevalência em 
mulheres não brancas, indicando, também, a necessidade de estudos voltados para a distribuição e análise da população, visto que diferentes regiões podem apresentar perfis epidemiológicos diferentes ${ }^{4}$.

Entretanto, vale ressaltar que em todos os casos, aqui analisados, a elevada incidência de sífilis congênita estava relacionada com alguma falha do sistema público de saúde em fornecer assistência pré-natal ideal para gestantes ${ }^{7,18,21}$.

\section{Fatores de influência.}

A análise dos dados nos estudos selecionados permitiu a observação de que a proporção de óbitos fetais entre os casos de sífilis congênita é seis vezes superior ao observado em mulheres sem o diagnóstico de sífilis na gestação, sendo visto, também, o elevado índice de internação dos recém-natos, podendo ser correlacionado com o tempo de exposição intrauterina do feto ao T. pallidum ${ }^{15,18}$.

Além disso, mulheres com menor escolaridade, que se autodeclararam negras ou pardas, atendidas em unidades públicas de saúde e em uso de drogas ilícitas, durante a gravidez, apresentaram maior possibilidade de desenvolver sífilis, associado a menor cobertura de exames para diagnóstico. A maioria das gestantes infectadas encontrou-se na fase latente da doença, quando, ainda, não se manifestaram os sintomas. Portanto, destaca-se a relevância do rastreamento da infecção empregando exames laboratoriais como os testes sorológicos e os testes rápidos ${ }^{18,27,28}$.

Ademais, mulheres com sífilis gestacional demonstraram maior índice de início tardio da assistência pré-natal, menor número de consultas pré-natal, realização de exames sorológicos e registro de sorologias reagentes no cartão de pré-natal ${ }^{15}$. Com base nesses dados, o aumento dos casos de sífilis congênita no Brasil pode ser atribuído ao cenário da sífilis adquirida na população brasileira em geral $^{17}$.

Por fim, vale pontuar que entre 2014 e 2017 houve um desabastecimento no Brasil e no mundo do principal antibiótico para tratamento de sífilis, a penicilina $G$ benzatina, procaína e cristalina ${ }^{29,30}$. À vista disso, os medicamentos disponíveis foram destinados prioritariamente ao tratamento de sífilis gestacional e de sífilis congênita. Contudo, observouse que mesmo com as reservas de medicamentos destinados para o tratamento prioritário de casos de sífilis congênita, houve um aumento exponencial durante esse período ${ }^{31}$. 


\section{CONSIDERAÇÕES FINAIS}

As análises dos dados apontam uma incidência de sífilis congênita elevada, ressaltando a necessidade da melhoria da qualidade da atenção à gestação e atenção à saúde, voltada para as populações mais vulneráveis.

Foi possível observar que houve aumento de sífilis gestacional e sífilis congênita na região Sudeste do Brasil. Destaca-se que os índices foram maiores na população de mulheres autodeclaradas pretas ou pardas, com baixa escolaridade, baixo índice socioeconômico e em uso de drogas ilícitas durante a gravidez. Portanto, ficou evidente a necessidade de estudos epidemiológicos regionais, para determinar o perfil populacional mais vulnerável em cada região.

Foi possível inferir a necessidade de aprimoramento dos profissionais da atenção básica, no intuito de assegurar um diagnóstico assertivo de sífilis e outras ISTs. Este ponto é indubitavelmente relevante, visto que pode influenciar positivamente o desfecho clínico, conduzindo ao tratamento adequado. Outra problemática evidenciada é atribuída à dificuldade na realização de testes laboratoriais confirmatórios, dado que são de difícil acesso, particularmente, no interior do Brasil, concomitantemente onde estão as regiões de maior vulnerabilidade. À vista disso, pesquisas que favorecem o diagnóstico da sífilis point-of-care serão de considerável valor, tanto para evitar gastos evitáveis à saúde pública, no tocante ao tratamento e cuidado, como, principalmente, na prevenção de danos à saúde da mãe e do filho.

Destarte, otimização dos serviços do pré-natal, campanhas informativas que atinjam os grupos mais vulneráveis, seguindo a identificação destes por região, são estratégias a serem consideradas.

Por fim, este estudo contribui com a discussão e reflexão de parâmetros relevantes para prevenção da sífilis congênita, que podem estar impactando no aumento da incidência de sífilis no Brasil. Os achados, aqui, discutidos podem ser úteis para auxiliar medidas de prevenção e atenção à saúde, ainda, podem contribuir para redução da incidência de novos casos, evitando os desfechos.

\section{AGRADECIMENTOS}

Os autores agradecem a Universidade de Brasília, ao Departamento de Farmácia e ao corpo docente do curso de Farmácia pelo apoio. 


\section{REFERÊNCIAS}

1. BRASIL, VIGILÂNCIA EM SAÚDE. Boletim Sífilis 2020 [Internet]. Out, 2020 [cited 2021 May 31]. Available from: http://www.aids.gov.br/pt-br/pub/2020/boletimsifilis-2020

2. PEELING R.W. et al. Syphilis. Nature Reviews Disease Primers [Internet]. 2017 Oct 12 [cited 2021 May 31];3(1):1-21. Available from: https://www.nature.com/articles/nrdp201773

3. BEZERRA M.L.M.B. et al. Congenital Syphilis as a Measure of Maternal and Child Healthcare, Brazil [Internet]. Vol. 25, Emerging Infectious Diseases. 2019. p. 1469-76. Available from: http://dx.doi.org/10.3201/eid2508.180298

4. MARINHO S.J. et al. Mother-to-child transmission and gestational syphilis: Spatialtemporal epidemiology and demographics in a Brazilian region. PLoS Negl Trop Dis [Internet]. $2019 \quad$ Feb;13(2):e0007122. Available from: http://dx.doi.org/10.1371/journal.pntd.0007122

5. BRASIL, MINISTÉRIO DA SAÚDE. Secretaria de Vigilância em Saúde Departamento de DST e HIV. Protocolo clínico e diretrizes terapêuticas para a prevenção da transmissão vertical do HIV, sífilis e hepatites virais. In: Ministério da Saúde. Secretaria de Vigilância em Saúde Departamento de DST e HIV, editor. Protocolo clínico e diretrizes terapêuticas para a prevenção da transmissão vertical do HIV, sífilis e hepatites virais [Internet]. 2015 [cited 2021 May 31]. Available from: http://www.aids.gov.br/pt-br/profissionais-de-saude/hiv/protocolos-clinicos-e-manuais

6. HORVÁTH A. Biology and Natural History of Syphilis [Internet]. Sexually Transmitted Infections and Sexually Transmitted Diseases. 2011. p. 129-41. Available from: http://dx.doi.org/10.1007/978-3-642-14663-3_11

7. COOPER J.M. SÁNCHEZ P.J. Congenital syphilis [Internet]. Vol. 42, Seminars in Perinatology. 2018. p. 176-84. Available from: http://dx.doi.org/10.1053/j.semperi.2018.02.005

8. ARAÚJO M.A.L. et al. Protocolo Brasileiro para Infecções Sexualmente Transmissíveis 2020: abordagem às pessoas com vida sexual ativa [Internet]. Vol. 30, Epidemiologia e Serviços de Saúde. 2021. Available from: http://dx.doi.org/10.1590/s1679-4974202100003.esp1

9. MACÊDO V.C. et al. Risk factors for syphilis in women: case-control study. Rev Saúde Pública [Internet]. 2017 Aug 17;51:78. Available from: http://dx.doi.org/10.11606/S1518-8787.2017051007066

10. RIBEIRO A.D.C. et al. Neurosyphilis in Brazilian newborns: a health problem that could be avoided. Rev Inst Med Trop Sao Paulo [Internet]. 2020 Nov 9;62:e82. Available from: http://dx.doi.org/10.1590/S1678-9946202062082 
11. MINISTÉRIO DA SAÚDE. Indicadores Sífilis - DCCI [Internet]. Indicadores e Dados Básicos da Sífilis nos Municípios Brasileiros. [cited 2021 May 31]. Available from: http://indicadoressifilis.aids.gov.br/

12. BRASIL, MINISTÉRIO DA SAÚDE. Secretaria de Vigilância em Saúde. Departamento de DST A e. HV. Protocolo Clínico e Diretrizes Terapêuticas para Atenção Integral às Pessoas com Infecções Sexualmente Transmissíveis (IST). Ministério da Saúde (BR). Secretaria de Vigilância em Saúde. Departamento de DST e HVPC. DTPAI as PCIST, editor. 2018.

13. KOJIMA N. KLAUSNER J.D. An Update on the Global Epidemiology of Syphilis. Curr Epidemiol Rep [Internet]. 2018 Mar;5(1):24-38. Available from: http://dx.doi.org/10.1007/s40471-018-0138-z

14. GALVÃO T.F. et al. Principais itens para relatar Revisões sistemáticas e Metaanálises: A recomendação PRISMA. Epidemiol Serv Saúde [Internet]. 2015 [cited 2021 May 31];24:335-42. Available from: http://www.scielo.br/scielo.php?script=sci_arttext\&pid=S2237-96222015000200335

15. DOMINGUES R.M.S.M. LEAL M do C. Incidência de sífilis congênita e fatores associados à transmissão vertical da sífilis: dados do estudo Nascer no Brasil. Cadernos de Saúde Pública [Internet]. 2016;32(6). Available from: https://www.scielo.br/scielo.php?pid=s0102$311 \times 2016000605002 \&$ script=sci_abstract\&tlng=es

16. MARQUES S.M. et al. Trends of syphilis in Brazil: A growth portrait of the treponemic epidemic. PLoS One [Internet]. 2020 Apr 9;15(4):e0231029. Available from: http://dx.doi.org/10.1371/journal.pone.0231029

17. OLIVEIRA V.S. et al. Aglomerados de alto risco e tendência temporal da sífilis congênita no Brasil. Rev Panam Salud Publica; 44, aug 2020 [Internet]. 2020; Available from: https://iris.paho.org/handle/10665.2/52524

18. BENEDETTI K.C.S.V. et al. High Prevalence of Syphilis and Inadequate Prenatal Care in Brazilian Pregnant Women: A Cross-Sectional Study. Am J Trop Med Hyg [Internet]. 2019 Oct;101(4):761-6. Available from: http://dx.doi.org/10.4269/ajtmh.180912

19. PIRES C.P. et al. Syphilis notifications among pregnant women in Campo Grande, state of Mato Grosso do Sul, Brazil, 2011 to 2017. Rev Soc Bras Med Trop [Internet]. 2020 Sep 11;53:e20200024. Available from: http://dx.doi.org/10.1590/0037-8682-00242020

20. ANDRADE A.L.M.B. et al. LATE DIAGNOSIS OF CONGENITAL SYPHILIS: A RECURRING REALITY IN WOMEN AND CHILDREN HEALTH CARE IN BRAZIL. Rev Paul Pediatr [Internet]. 2018 Jul;36(3):376-81. Available from: http://dx.doi.org/10.1590/1984-0462/;2018;36;3;00011 
21. MARTINEZ E.Z. et al. Attributable fraction of congenital syphilis due to the lack of prenatal care. Rev Soc Bras Med Trop [Internet]. 2019 Jul 18;52:e20180532. Available from: http://dx.doi.org/10.1590/0037-8682-0532-2018

22. RAC M.W.F. et al. Syphilis during pregnancy: a preventable threat to maternal-fetal health. Am J Obstet Gynecol [Internet]. 2017 Apr;216(4):352-63. Available from: http://dx.doi.org/10.1016/j.ajog.2016.11.1052

23. CASAL C.A.D. et al. Molecular detection of Treponema pallidum sp. pallidum in blood samples of VDRL-seroreactive women with lethal pregnancy outcomes: a retrospective observational study in northern Brazil. Rev Soc Bras Med Trop [Internet]. 2011 Jul;44(4):451-6. Available from: http://dx.doi.org/10.1590/s003786822011005000047

24. OLIVEIRA D de. et al. Rollet's mixed chancre in Brazilian pregnant women: An unusual case report [Internet]. Vol. 91, International Journal of Infectious Diseases. 2020. p. 57-9. Available from: http://dx.doi.org/10.1016/j.ijid.2019.11.011

25. DOMINGUES R.M.S.M. et al. Prevalence of syphilis in pregnancy and prenatal syphilis testing in Brazil: birth in Brazil study. Rev Saude Publica [Internet]. 2014 Oct;48(5):766-74. Available from: http://dx.doi.org/10.1590/s00348910.2014048005114

26. TUSSET D. et al. Programa Academia da Saúde: correlação entre internações por doenças crônicas não transmissíveis e adesão nos municípios brasileiros, 2011-2017 [Internet]. Vol. 29, Epidemiologia e Serviços de Saúde. 2020. Available from: http://dx.doi.org/10.1590/s1679-49742020000500013

27. ACOSTA L.M.W. et al. Coinfecção HIV/sífilis na gestação e transmissão vertical do HIV: um estudo a partir de dados da vigilância epidemiológica. Rev Panam Salud Publica [Internet]. 2016 Dec [cited 2021 May 31];40:435-42. Available from: https://www.scielosp.org/article/rpsp/2016.v40n6/435-442

28. BRASIL, MINISTÉRIO DA SAÚDE. Secretaria de Ciência. IE-C. Protocolo clínico e diretrizes terapêuticas para atenção integral às pessoas com infecções sexualmente transmissíveis. da Saúde M, editor. 2015.

29. Desabastecimento de penicilina alerta para desafio global de combate à sífilis [Internet]. 2016 [cited 2021 Aug 10]. Available from: https://portugues.medscape.com/verartigo/6500488

30. ÁVILA A.P.O. MELO KCC de. Separação de poderes e diálogos institucionais nos litígios envolvendo o direito à saúde. Rev Investig Const [Internet]. 2018 Jan 1;5(1):83. Available from: http://revistas.ufpr.br/rinc/article/view/54934

31. ARAUJO R.S. et al. Who was affected by the shortage of penicillin for syphilis in Rio de Janeiro, 2013-2017? Rev Saude Publica [Internet]. 2020 Nov 2;54:109. Available from: http://dx.doi.org/10.11606/s1518-8787.2020054002196. 